\title{
Innovations sociales et rationalisation budgétaire : quelles interactions entre deux logiques institutionnelles antagonistes?
}

Social innovation and budget rationalization: which interactions between two opposing institional logics?

Patrick Gilbert, Nathalie Raulet-Croset et Carine Chemin-Bouzir

\section{OpenEdition \\ Journals}

Édition électronique

URL : http://journals.openedition.org/pmp/5765

ISSN : 2119-4831

Éditeur

Institut de Management Public (IDPM)

\section{Édition imprimée}

Date de publication : 15 décembre 2012

Pagination : 607-627

ISBN : 978-2-7430-1492-6

ISSN : 0758-1726

Référence électronique

Patrick Gilbert, Nathalie Raulet-Croset et Carine Chemin-Bouzir, «Innovations sociales et rationalisation budgétaire : quelles interactions entre deux logiques institutionnelles antagonistes ?», Politiques et management public [En ligne], Vol 29/4 | 2012, mis en ligne le 26 février 2015, consulté le 02 mai 2019. URL : http://journals.openedition.org/pmp/5765 


\title{
Innovations sociales et rationalisation budgétaire : quelles interactions entre deux logiques institutionnelles antagonistes?
}

\author{
$>$ Patrick Gilbert ${ }^{a}$, Nathalie Raulet-Croset ${ }^{b}$ \\ et Carine Chemin-Bouzir ${ }^{* *}$
}

a Professeur (Gregor, IAE de Paris), IAE de Paris - 21 rue Broca - 75240 Paris Cedex 05

${ }^{b}$ Maître de conférences (PREG-CRG École Polytechnique et IAE de Paris)

IAE de Paris - 21 rue Broca - 75240 Paris Cedex 05

c Professeur permanent, Reims management school (chercheure associée au GREGOR

49, rue Pierre Taittinger - 51100 Reims

\section{Résumé}

Dans le champ de l'action sociale, s'opposent deux logiques institutionnelles, celle de l'innovation et celle de la rationalisation budgétaire. Elles semblent de prime abord inconciliables. La première, porteuse de diversité et d'idiosyncrasie, serait l'apanage des associations. La seconde donnerait la part belle aux financeurs publics en visant I'homogénéisation et la transparence de l'action associative. Le caractère irréductible de cette opposition dualiste est-il avéré ? L'article étudie les interactions entre les deux logiques à travers l'étude du cas d'une association et des modalités de son financement. Il s'attache, tout d'abord, à décrire les formes concrètes des relations association-financeurs dans le cas étudié. Puis, il analyse les processus temporels et multi-acteurs, montrant le dépassement de l'apparente incompatibilité entre les deux logiques.

(c) 2012 IDMP/Lavoisier SAS. Tous droits réservés

Mots clés : logique institutionnelle, innovation sociale, financement, dispositif de gestion, action sociale.

\section{Abstract}

Social innovation and budget rationalization: which interactions between two opposing institutional logics. In the field of social action, there are two opposing institutional logics: innovation and budget rationalization. At first sight they appear irreconcilable. The first, valuing diversity and idiosyncrasy would seem to be the prerogative of non-profit organisations. The second would seem to be given pride of place by public funding, aiming at homogenisation and transparency in social action. But is the outright opposition between these

*Auteur correspondant : carine.chemin-bouzir@reims-ms.fr doi:10.3166/pmp.29.607-627 @ 2012 IDMP/Lavoisier SAS. Tous droits réservés 
two ideas insurmountable? This article studies interaction between the two logics through the case study of a non-profit organisation and the methods of its funding. First it describes the different types of relationship between the organisation and its funders. Then it analyses the temporal and multi-player processes, showing how the apparent incompatibility between the two logics can be overcome.

(c) 2012 IDMP/Lavoisier SAS. Tous droits réservés

Keywords: institutional logic, social innovation, financing, management tool, social action.

\section{Introduction}

Entre les préoccupations financières et l'innovation sociale, qui mobilisent, l'une et l'autre, les acteurs associatifs, il semble de prime abord y avoir un large gouffre. Pour le sens commun, finance et innovation habitent des mondes rivaux. Leur opposition serait aujourd'hui exacerbée, car en période de raréfaction des financements, les financeurs ont acquis un rôle nouveau. Ils mettent en valeur certaines activités, en découragent d'autres, à travers l'octroi de budgets et la définition de conditions et de critères.

À la perspective supposée instrumentale et néo-déterministe des financeurs s'oppose, comme une évidence, l'innovation sociale, en tant qu'elle est faite d'émergences (Klein et Harrisson, 2007), d'approximations, de tâtonnements, d'essais-erreurs. Certains travaux sociologiques semblent accréditer cette thèse. Ainsi, s'inspirant de la conception de l'innovation établie par Merton (1949/1965), Norbert Alter (2000, 2006) soutient-il que l' « innovation ordinaire » implique une transgression de l'ordre établi, une destruction des règles en usage. Dans cette perspective, la conformité des activités de l'association aux attentes, surplombantes, des financeurs, aurait pour conséquence des effets dévastateurs, inhibant la créativité des acteurs sociaux. Les dirigeants d'association n'auraient donc d'autre alternative que d'épouser la logique financière, en vue de s'y aligner - de s'y soumettre -, au risque de s'enfermer dans des activités familières, bien repérées par les financeurs, ou de la transgresser afin de continuer à innover. Est-ce bien sûr?

La légitimité d'une association, au sens de Meyer et Rowan (1977) et Di Maggio et Powell (1983), et son audience auprès des financeurs, ne pourraient-elles pas provenir au moins autant de son statut d'innovateur social que de sa capacité à respecter les normes que véhiculent les dispositifs de financement? Aussi, à une posture de dépendance aux exigences de rationalisation, se confronterait une logique d'innovation sociale, souvent liée au projet qui donne sens à l'activité associative, cherchant à faire bouger les lignes, à bousculer les normes existantes dans un contexte donné (Chambon, David et Devevey, 1982). Aussi la conduite d'une association est-elle fortement influencée à la fois par sa dynamique en termes d'innovation sociale, et par les nouveaux processus de financement public. Mais ces dynamiques sont-elles en opposition, ou existe-t-il entre elles des formes d'interaction vertueuses?

C'est sur la base de ces premières réflexions que s'est tissée notre question de recherche : comment dispositifs de financement et innovation sociale s'articulent-ils? Notre objectif dans cet article est d'approfondir l'hypothèse selon laquelle on peut dépasser l'opposition 
apparente entre dynamiques d'innovation et contraintes de financement. Après une analyse du contexte des financements, nous montrons, à la lumière d'une étude de cas, que l'on ne peut s'en tenir à l'hypothèse de sens commun selon laquelle les financeurs seraient porteurs d'une forme de régulation de contrôle (Reynaud 1988), bloquant les potentialités d'innovation, alors que les associations seraient au contraire porteuses de logiques d'innovation dans le cadre d'une régulation autonome. En effet, si certaines orientations de financement peuvent apparaître comme des freins, d'autres offrent un cadre favorable à l'innovation.

\section{Les transformations du contexte des financements}

\subsection{La politique de la ville : d'un relâchement de la contrainte, au retour de la rigueur budgétaire}

La « rationalisation des choix budgétaires », installée à la fin des années 1960, était étroitement centrée sur des études coût-efficacité. Prenant son contre-pied, dans les années 1970 et 1980, les programmes « Habitat et vie sociale » et « Développement social des quartiers » mettent en place une nouvelle politique d'action publique dans des quartiers d'habitat social, faite de transversalité, de partenariat et de participation des habitants (Epstein, 2010). Un nouveau type de politique publique et un nouveau type de gouvernance de cette politique associent les associations locales et doivent permettre l'expérimentation (Heyraud, 2010). Les années suivantes voient des lignes budgétaires propres à la politique de la Ville se créer et être abondées. Elles sont mobilisées dans le cadre de « contrats de Ville » signés en 1994 puis 2000 entre l'État et les collectivités territoriales concernées. Le dispositif évoluera sous l'appellation Contrat urbain de cohésion sociale (CUCS) en 2006.

Les critiques de cette politique de la part des tenants d'une gestion rigoureuse des deniers publics se font particulièrement entendre à partir du début des années 2000. En 2002, un rapport de la Cour des comptes mentionne «le défaut d'objectifs nationaux opérationnels et stables, assortis de calendriers d'actions et de résultats ». La loi d'orientation et de programmation pour la politique de la Ville et la rénovation urbaine du $1^{\text {er }}$ août 2003 tente de répondre à ces observations. En novembre 2007 pourtant, la Cour des comptes réitère ses critiques dans un nouveau rapport. Lors des consultations menées pour l'élaboration de ce rapport, la direction du budget de l'État indique sa préférence pour une structure administrative pilote au niveau national et d'opérateurs qui déclinent cette politique nationale. Il convient dans cette optique que «l'opérateur ne soit pas livré à trop de choix possibles dans la manière de décliner la politique » (Cour des Comptes, 2007, p 40) et que des contrats d'objectifs et de performance assortis d'indicateurs s'assurent du respect de ces objectifs.

\subsection{Une évolution similaire de l'action sociale des Caf}

L'action sociale, dans la traduction qu'en donnent les Caisses d'allocations familiales (Caf), financeurs de nombre de structures associatives, connaît une évolution similaire. Alors que ces organismes sont historiquement des organismes à la gouvernance locale associant les syndicats et les associations familiales locales, le Plan Juppé de 1995-1996 donne un rôle plus important au Parlement et à l'État en matière de financement de la sécurité sociale. 
Des conventions d'objectifs et de gestion (COG) pluriannuelles sont instaurées entre l'État et chaque branche de la Sécurité sociale (Damon, 2008). En conséquence, la Caisse nationale des allocations familiales, qui était une tête de réseau, devient l'animateur de la mise en œuvre des COG, en déclinant la politique nationale au niveau local dans une visée de rationalisation de la dépense publique, tout en laissant une petite autonomie financière aux Caf pour soutenir des innovations locales.

Les transformations de la politique de la ville comme celles de l'action sociale induisent aujourd'hui de fortes contraintes de financement et suscitent des tensions entre les financeurs et les associations locales. Demoustier (2005) observe que ces dernières craignent de devenir de simples courroies de transmission des dispositifs publics et se rebellent contre le risque d'instrumentalisation qu'elles perçoivent. Pourtant, ainsi que le relève cet auteur, ces deux acteurs ne sont-ils pas condamnés à vivre ensemble ? $\mathrm{Ne}$ doivent-ils pas pour retrouver une dynamique de progrès social s'efforcer de conjuguer des logiques en confrontation?

\subsection{Deux logiques en confrontation}

Ces formes de relations entre associations et pouvoirs publics s'organisent entre deux logiques qui coexistent, que nous nommons logique de l'innovation sociale et logique de la rationalisation budgétaire. Alors que le point précédent retraçait l'émergence historique de ces deux logiques, nous proposons maintenant de pointer leurs différences.

Tout d'abord, sans être les attributs de tel ou tel type d'acteur, elles donnent chacune la primeur à l'un des acteurs dans l'initiative et la définition des pratiques à mettre en place. Alors que la logique de la rationalisation budgétaire voudrait que des priorités soient fixées au niveau national et déclinées par des opérateurs dotés d'une feuille de route, la logique de l'innovation sociale donne la part belle aux associations et aux citoyens : l'innovation sociale vient du terrain (Lévesque 2002), la reconnaissance institutionnelle venant dans un second temps (Chambon et al., 1982).

Ensuite, les dynamiques sont également différentes en matière d'homogénéité ou de diversité de l'offre. La logique de rationalisation budgétaire voudrait que, pour rendre les actions locales comparables, elles soient la déclinaison d'une politique homogène à l'échelle territoriale du financeur. Au contraire, la logique de l'innovation, qui apparaît sous le vocable d' « expérimentation » admet la diversité des réponses locales ou micro-locales, en fonction d'une configuration d'acteurs et de l'identification de besoins spécifiques. L'innovation est une « discontinuité par rapport aux pratiques habituellement mises en œuvre dans un milieu donné » (Cloutier, 2003 : 8) qui répond aux lacunes dans les institutions socio-politiques (Hillier, Moulaert et Nussbaumer, 2004 : 135).

Enfin, la différence est grande entre la logique de rationalisation budgétaire qui vise à rendre l'action lisible, et la logique de l'innovation par laquelle on lance des actions dont personne ne peut savoir ex ante quels en seront les effets. Les évaluations se heurtent pour mesurer les impacts des politiques publiques au manque de cohérence que les diversités locales génèrent et il en ressort au niveau national l'impression d'un « saupoudrage » qui rend l'action inefficace. La logique de l'innovation s'inscrit dans une visée différente, où il conviendrait parfois d'expérimenter dans l'ombre avant de faire savoir ce qui est réalisé. 


\section{La coexistence de deux logiques institutionnelles}

Les deux logiques identifiées, celle de la rationalisation budgétaire et celle de l'innovation sociale, peuvent être qualifiées de logiques « institutionnelles », dans la perspective de la théorie néo-institutionnaliste, car elles sont soutenues par des acteurs institutionnels et sont porteuses de valeurs et pratiques spécifiques. Différents auteurs mobilisent le concept de « logique institutionnelle » (Friedland et Alford 1991, Thornton et Ocasio, 1999) pour désigner un ensemble de croyances, valeurs, outils, et pratiques, qui sont portés par des institutions et qui structurent les actions organisationnelles et individuelles.

Les développements récents des travaux sur les logiques institutionnelles s'intéressent à l'existence de logiques contradictoires qui coexistent sur la durée au sein d'un même champ organisationnel (au sens de Di Maggio et Powell, 1983), et analysent leurs effets sur les organisations (Reay et Hinings 2009). Face à cette complexité institutionnelle (Greenwood et al., 2011), les organisations développent en effet des réponses organisationnelles multiples. Certaines produisent une différenciation des unités organisationnelles permettant de concilier les différentes logiques (Jarzabkowski, Matthiesen et Van de Ven, 2010), d'autres construisent progressivement une identité mêlant les logiques institutionnelles et deviennent des organisations dites « hybrides » (Battilana et Dorado, 2010). D'autres recherches s'intéressent aux processus politiques intra-organisationnels et à la façon dont certains groupes dans une organisation peuvent soutenir les demandes liées aux différentes logiques institutionnelles (Pache et Santos, 2010).

Le champ de l'intervention sociale, présenté précédemment, peut être qualifié de fragmenté (Pache et Santos 2010) au sens où il n'existe pas d'unité dans le champ, mais un ensemble d'acteurs non coordonnés, qui soutiennent en partie les deux logiques, y sont confrontés, aussi bien du côté des financeurs que des acteurs de l'économie sociale et solidaire. L'incompatibilité entre les logiques est selon Pache et Santos (2010) d'autant plus forte si les idéologies qui les sous-tendent sont incompatibles; elles peuvent également s'opposer au regard des moyens et actions qu'elles prescrivent. Le degré d'ambiguïté (Goodrick et Salancick, 1996; Greenwood et al., 2011) quant aux prescriptions d'une logique, joue également sur la possibilité pour un acteur d'échapper à l'emprise d'une logique institutionnelle. Dans le cas du champ de l'action sociale, les logiques de rationalisation budgétaire et d'innovation ne s'opposent pas sur le plan des buts et des idéaux, mais comme nous l'avons montré sur le plan de l'organisation de l'action et des moyens mis en place. Par ailleurs, la coexistence sur le long terme, et la multiplicité des acteurs porteurs de l'une, de l'autre ou des deux logiques, semble permettre une certaine ambiguïté et donc un degré de latitude pour les acteurs du champ organisationnel.

Il est alors intéressant de se demander comment la logique de rationalisation budgétaire s'articule avec la logique d'innovation sociale dans le champ organisationnel de l'intervention sociale. Elles s'opposent sur plusieurs points, et peuvent de ce fait pour certains acteurs conduire à l'exclusion de la logique d'innovation au profit de la logique de rationalisation budgétaire. Mais elles peuvent aussi cohabiter. Comment la coexistence entre ces deux logiques peut-elle se produire? Quels sont les facteurs qui permettent, pour un acteur donné, cette cohabitation? 


\section{Méthode : une étude de cas à visée descriptive et compréhensive}

Pour répondre aux questions formulées, nous souhaitions avoir accès à une compréhension approfondie du lien entre innovations sociales et dispositifs de financement et décrire ce phénomène dans toute sa complexité. Selon les principes établis de longue date par la recherche qualitative en sciences sociales (Hamel, 1997), nous avons réalisé une étude de cas exploratoire, au sens de Yin (1990). Centrée sur la situation étudiée, cette étude de cas a essentiellement une visée descriptive et compréhensive.

Notre terrain est Lien et Proximité (nom fictif), une association de l'Est de la France œuvrant pour la cohésion sociale, et qui est reconnue régionalement comme légitime par les autres acteurs sur son champ. Cette association, choisie pour sa réputation d'innovateur social, au niveau local, est comme beaucoup dans son secteur d'activité, fortement dépendante des financements publics, car son objet est d'utilité sociale et son public habituel peu solvable.

Le recueil de données a été réalisé entre novembre et décembre $2009^{1}$. Il combine trente entretiens individuels semi-directifs, auprès des acteurs associatifs et des financeurs, quatre observations-participantes sur les deux principales activités de l'association et l'étude de documents internes (budgets, rapports d'activité, demandes de financement..). Les données recueillies ont fait l'objet de comptes rendus détaillés (pour les observations), d'enregistrements et de retranscriptions (pour les entretiens). Elles ont été analysées dans la perspective développée par Glaser et Strauss (1967).

L'analyse a permis de caractériser les principaux financeurs du point de vue des finalités poursuivies et des modalités et critères de financement. Elle montre également comment les acteurs associatifs interagissent avec les dispositifs de financement et quel lien les démarches et actions de financement entretiennent avec l'innovation.

\section{Lien et Proximité : le cas d'une association innovante face à de nouvelles logiques financières}

\subsection{Présentation de l'association}

Deux orientations majeures fondent la raison d'être de l'association Lien et Proximité : il s'agit à la fois de susciter le «contact, comme rencontre de personnes d'origines, de milieux, d'âges... différents, pour s'ouvrir aux autres et partager» et de «contribuer au développement des compétences, [pour] diminuer le poids des inégalités » (Projet associatif de Lien et Proximité), en particulier entre des personnes qui seraient peu amenées à se rencontrer, à savoir le public de l'association et les bénévoles qui travaillent en son sein. L'importance du bénévolat constitue en effet une spécificité de l'association. Les activités s'orientent autour de l'accueil des familles, du travail avec l'enfant dans sa globalité, et visent à « s'inscrire principalement dans les interstices non couverts par les dispositifs institutionnels légaux ou réglementaires » (Projet associatif de Lien et Proximité).

L'insertion des acteurs associatifs dans les réseaux locaux permet de faire connaître et reconnaître ses activités. Le Président de l'association notamment indique qu'il a été

\footnotetext{
${ }^{1}$ Pour plus de précisions, consulter la « Note méthodologique » figurant en annexe.
} 
pendant douze ans administrateur d'un centre culturel, puis qu'il a eu des responsabilités à la Croix Rouge. Le Maire adjoint en charge du quartier souligne qu'il « est clair aussi que la directrice et le président sont des éléments importants » pour s'assurer du bien-fondé de l'association, et que «sur la ville, c'est facile de les défendre, ils sont connus. » le Directeur de l'action sociale de la Ville confirme : "nous connaissons Lien et Proximité par des contacts terrain. » Dans le contexte particulier de difficultés financières, «ils ont très bien géré le relationnel avec les institutionnels puisqu'ils sont allés voir tout le monde.» (Chargé de mission politique de la Ville Préfecture).

Forte de ces atouts, Lien et Proximité a su se faire reconnaître localement comme ayant mis en place des activités innovantes. Parmi ces activités, nous avons observé spécialement trois d'entre elles, choisies en raison, d'une part, du poids élevé que représente leur financement dans les ressources de l'association et, d'autre part, de leur caractère contrasté du point de vue de l'innovation :

- Une première activité radicalement nouvelle dans son contenu, la « Parlothèque », un lieu d'accueil associant les parents et leurs enfants, alors que les services proposés à ces publics étaient jusqu'ici différenciés.

- Une deuxième activité, les cours de Français Langue Étrangère (FLE) à visée sociale, qui consiste en un apprentissage de la langue dans un objectif de facilitation d'insertion sociale. Son originalité réside dans son processus de réalisation. Loin de rester dans une relation classique entre professeur et apprenant, les séances créent, autour de la relation d'apprentissage, des liens entre le public d'apprenants et d'autres acteurs de la vie locale, qu'il s'agisse des enseignants qui, bénévoles pour la plupart, vont souvent au-delà de la simple relation d'apprentissage de la langue, ou qu'il s'agisse d'autres acteurs du territoire local.

- Une troisième activité, le FLE à visée professionnelle, qui peut être lue comme une diversification de la précédente. Elle s'en distingue par un changement de visée. L'objectif est ici de faciliter aux personnes l'accès au marché du travail. Elle connaît actuellement un certain essor dans l'association car elle devient l'objet de financements spécifiques.

Au-delà de l'opposition apparente entre ces différentes activités, elles se rejoignent sur leur conception de la création de liens entre des publics qui, d'ordinaire, sont traités séparément. Nous mobilisons pour l'analyse de ces activités la distinction classique, en management stratégique, entre innovation de rupture (ou innovation radicale) et innovation incrémentale, qui nous permettra de caractériser les activités observées.

\subsection{Innovation de rupture, innovation incrémentale}

L'innovation de rupture qui implique une transformation des comportements est la plus spectaculaire. Elle se démarque des traditions et des conventions et repose sur la mise en œuvre de compétences nouvelles. Cependant, les véritables innovations de rupture sont rares. Il est extrêmement difficile de remettre en cause des conventions ancrées dans les représentations, mais aussi et surtout dans des dispositifs, des institutions, des circuits de communication, des modèles de relations, etc. Moins directement perceptible, l'innovation incrémentale s'appuie sur les conventions existantes. Elle ne cherche pas à les remettre en cause mais seulement à apporter une variation à la marge. Cette démarche permet ainsi 
d'approfondir un besoin en trouvant de nouveaux bénéficiaires qui sont intéressés par les améliorations, parfois élémentaires, qui sont apportées. Elle peut très bien exister au sein d'activités quotidiennes au cours desquelles des acteurs interprètent les situations en constatant un écart entre l'existant et le souhaitable, et pour autant qu'ils en aient la capacité, cherchent à infléchir le cours des événements. Elle se produit de manière régulière et progressive au travers de changements résultant de processus d'apprentissage collectif.

Dans la vie d'une organisation, on voit se succéder de longues périodes de progression incrémentale suivant d'exceptionnelles innovations radicales (Tushman et Romanelli, 1985; Tushman et Anderson, 1986; Atamer, Durand, Reynaud, 2005); d'autres auteurs montrent l'importance de la combinaison et des apports réciproques entre innovation d'exploitation, à caractère incrémental, et les innovations d'exploration, à caractère plus radical (Grève, 2007 ; Chanal et Mothe, 2005). Cette distinction entre les deux types d'innovation déborde largement le monde industriel où elle est née. Elle nous paraît intéressante pour distinguer, au regard des « histoires d'innovation » que nous avons reconstituées, leur lien avec les dynamiques de financement.

\subsection{La Parlothèque : une innovation de rupture, créatrice de légitimité}

\section{Une activité hors-norme}

La Parlothèque réalise un ensemble diversifié d'activités : accueil des parents avec leurs enfants tous les jours; création de supports à la conversation à la socialisation et à l'épanouissement des familles dans ses propres locaux et « hors les murs » : ateliers conte/ cuisine/arts plastiques/musique; organisation d'événements (fêtes, anniversaires, Nouvel An du pays d'origine, etc.); interventions hebdomadaires à la PMI (Protection maternelle et infantile) et à la médiathèque, médiation avec les enseignants.

La désignation comme «innovation de rupture » renvoie au caractère « hors norme » du projet : il n'existait pas à l'époque où la Parlothèque a été créée une telle conception de la relation parents-enfants, ainsi que le soulignent les coupures de presse que nous avons examinées et les témoignages des administrateurs comme ceux des financeurs. En effet, les rares antécédents qui existent, sur d'autres territoires, répondent à une logique d'action différente. Dans les années 1970, des lieux d'accueil sont créés sous l'égide de psychanalystes à Paris. Notamment, la « Maison Verte » de Françoise Dolto est créée en 1979 et elle sera à l'origine de la création de quelques autres structures de la même appellation. Au début des années 1990, les rares initiatives autour de « lieux d'accueil nouveaux pour les jeunes enfants » s'inscrivent dans cette lignée psychanalytique (voir par exemple Neyrand, 1995).

La Parlothèque se distingue de cette approche psychanalytique par son intention éducative, résumée par la formule «papoter autour d'un faire ». Elle promeut, vis-à-vis des parents et des enfants, des activités ludiques communes aux parents et aux enfants accueillis qui sont le support à un soutien de type éducatif, et non psychologique.

\section{Un financement qui ne va pas de soi}

Localement, en ce qui concerne la Parlothèque, la réflexion a démarré en 1990, sur un quartier populaire que l'association connaissait bien, puisqu'elle y était impliquée depuis une quinzaine d'années, à travers des activités périscolaires et des cours de FLE. Le montage financier du projet a été laborieux, notamment pour ce qui concerne le choix du lieu d'implantation et la construction des locaux (un local de $150 \mathrm{~m}^{2}$ ). La difficulté majeure 
du point de vue du financement est que ce projet ne correspondait à aucune des catégories de financement prévues : «il n'entrait dans aucun tiroir » (Directeur action sociale Caf).

Du côté des financeurs, la Cnaf prend acte de l'émergence des lieux d'accueil enfantsparents en 1996. Elle inscrit le soutien à la fonction parentale dans ses orientations d'action sociale en 1997. En 2002, elle diffuse une circulaire dans laquelle elle précise ce qu'elle entend par lieu d'accueil enfants-parents (LAEP), et ouvre droit à des financements pour les structures qui répondent à son cahier des charges. La circulaire qui précise ce que doivent être les LAEP s'inscrit toujours dans l'approche psychanalytique des débuts du mouvement. Elle précise que les accueillants doivent être «formés à l'écoute », et prévoit une supervision psychologique ou psychanalytique.

$\mathrm{Au}$ moment de la création de la Parlothèque, l'institutionnalisation des lieux enfantsparents n'a pas encore eu lieu, et les années suivantes cette institutionnalisation promeut une action qui ne correspond pas à l'orientation éducative de la Parlothèque. Cela explique pourquoi les conditions du montage financier ont été difficiles, mais aussi pourquoi la Parlothèque se démarque aujourd'hui de ce que la Caf entend par « lieu d'accueil enfants parents ». Les activités supports développées par la Parlothèque sont en conséquence considérées comme une déviance par la Caf.

La Parlothèque nous paraît donc plus directement relever de l'innovation de rupture, en raison de changements significatifs pour l'ensemble des acteurs impliqués. Toutefois, il y a dans la naissance de cette activité des éléments qui la placent dans une progression : elle est autant le fruit de l'expérience que de l'invention. Réalisé en 1996, le bilan d'une première expérimentation fait référence à plus de vingt ans d'expérience en matière d'activités périscolaires auprès d'enfants en difficulté et d'alphabétisation auprès de familles immigrées qui ont précédé la création de cette activité. Avec le temps, la Parlothèque est également apparue comme une innovation qui a été prolongée par d'autres, plus incrémentales. Des activités sont développées en interne, comme « les goûters équilibrés » ou les « moments musicaux ». Des actions sont développées à la bibliothèque, dans les écoles, lors de sorties organisées par la Parlothèque. En interne comme « hors les murs », le « faire » est un support à la discussion.

La Ville, principal financeur de la Parlothèque (plus de $80 \%$ du budget en 2009), reconnaît volontiers le caractère novateur de la Parlothèque et valorise son originalité et son rayonnement :

«La [Parlothèque] est un lieu d'accueil Parentlenfant assez spécifique et qui a été à sa création, quelque chose d'assez emblématique dans une démarche originale et construite de façon intelligente et avec rigueur, notamment dans l'approche des Parents et des enfants et qui a même été un modèle. Je sais qu'on venait d'autres villes voir quand [Lien et Proximité] a monté cette [Parlothèque]. Ça reste un endroit, [dans ce quartier], qui est un endroit important pour le travail avec les familles et qui est soutenu, d'autre part. »

(Responsable du service Enfance de la Ville).

La Caf, deuxième financeur, reconnaît aussi le caractère innovant de la Parlothèque à son origine : «c'était une innovation à l'époque » (Directeur action sociale Caf). Mais elle a des difficultés à articuler la demande d'homogénéisation qui vient du niveau national et la dynamique d'innovation locale portée par Lien et Proximité : "C'était une innovation sur laquelle nous avons estampillé LAEP après (...) ça devient une offre de service si on peut dire sous cet angle là. » La volonté est de «maîtriser» «l'opérateur de politique publique » qu'est devenu à ses yeux Lien et Proximité. 
Face à cette volonté de maîtrise de l'activité revendiquée par la Caf, Lien et Proximité mobilise parfois des financements complémentaires. Par exemple, elle a financé une activité de conte à la médiathèque, appelée « raconte tapis » dans le cadre d'un dispositif géré par un service d'État auquel la Caf abonde, à hauteur de 12000 euros environ en 2007 et 2008. Les représentants de la Caf, découvrant cette activité a posteriori, ont le sentiment que l'association se disperse, et contestent cette politique. Ils ne démentiront cependant pas leur soutien financier les années suivantes.

Le cas de la Parlothèque montre que la logique institutionnelle d'innovation structure les croyances et les comportements non seulement des associations, mais aussi des financeurs publics. Innovation reconnue, la Parlothèque est en effet soutenue de ce fait par la Mairie et par ricochet, par la Caf. Cette légitimité acquise permet par la suite de maintenir un projet original et de le développer, nonobstant les contraintes de rationalisation budgétaire et en particulier d'homogénéisation de l'offre nationale affichées par la Caf.

\subsection{Le FLE à visée sociale : une trajectoire d'innovations incrémentales}

\section{Une activité classique dans son contenu, innovante par son processus}

Sur le contenu, les actions de FLE, mises en œuvre dès la création de l'association, semblent n'avoir rien de spécifiquement novateur. De nombreux organismes proposent ce service d'apprentissage de la langue étrangère. "les structures de proximité [dans la Région] proposaient une offre de formation beaucoup plus dense que dans bon nombre de régions avec un volume budgétaire beaucoup plus important. » (Chargée de mission centre de ressources pour les formations linguistiques missionnées par la Ville). Toutefois, chez Lien et Proximité, le FLE prend un visage spécifique, car l'apprentissage est réalisé au sein de petits groupes, avec la présence d'une majorité de bénévoles dans l'équipe. Les témoignages d'apprenants et de bénévoles montrent que les relations intenses qui s'instaurent entre les différents participants aux ateliers atteignent la dimension d'insertion sociale. Le FLE chez Lien et Proximité se distingue des pratiques existant ailleurs, car il s'appuie sur une relation bénévole-apprenant qui mêle apprentissage de la langue et apprentissage social.

Si le FLE à visée sociale ne constitue pas une innovation de rupture, dans la mesure où il s'insère dans un ensemble de pratiques existantes relativement similaires, ses modalités de mise en œuvre chez Lien et Proximité s'inscrivent dans la recherche de liens de nature nouvelle entre acteurs pour mieux appréhender l'environnement social, et comportent de ce fait une dimension d'innovation sociale.Ainsi, des sorties sont organisées pour accroître l'insertion sociale et pratiquer la langue en situation. L'apprentissage de l'usage de l'ordinateur est introduit, pour familiariser les apprenantes à cet outil important de la vie quotidienne, certaines mamans pouvant alors mieux communiquer autour de l'outil avec leurs enfants, etc... Ces différentes actions renvoient à des formes d'innovation incrémentale, qui contribuent à modeler l'activité de FLE à visée sociale.

Une structure de financement composite

Comme la Parlothèque, l'activité de FLE à visée sociale est financée par des versements stables et « lourds » pour son activité principale et par des financements spécifiques pour les projets d'innovation incrémentale. À la différence de la Parlothèque, toutefois, le financement est plus éclaté et le poids relatif des financeurs (État, Ville, Union Européenne, Conseil général, Préfecture...) évolue au fil du temps, créant un élément d'incertitude quant aux revenus de l'Association. 
Les actions périphériques ou innovations incrémentales sont financées par des budgets spécifiques. Le « FLE informatique » nécessitait l'achat de micro-ordinateurs, ce qui a été rendu possible par des subventions d'investissement de la Fondation Orange, de 933 euros en 2008 et de 2369 euros en 2009. Le « FLE été jeunes » a été financé en 2008 à hauteur de 1000 euros par le Conseil général et de 1130 euros par l'ACSE (agence nationale pour la cohésion sociale et l'égalité des chances). Enfin, la réponse à un appel d'offres concernant la lutte contre l'illettrisme a rendu possible un financement de 10000 euros de la politique de la Ville État.

\section{Un financeur qui change son orientation}

L'action historique de cours de Français pour adulte est traditionnellement financée dans le cadre de la politique de la Ville. Dans la Région, le Fonds Social Européen (FSE), en principe dédié à l'emploi et au développement économique, a en outre historiquement accepté une compréhension large de ses objectifs. Toutefois, le protocole 2007-2013 du FSE resserre les objectifs sur l'insertion professionnelle, et le FLE à visée sociale ne sera plus financé par le FSE à partir de 2008. La perte représente plus de 28000 euros (soit $55 \%$ du budget de l'action) pour l'association Lien et Proximité.

Au-delà de Lien et Proximité, la perte financière touche différentes associations qui font savoir que leur activité est mise en péril et adressent à la Ville des demandes de subventions plus importantes et plus nombreuses. L'élue en charge de l'action sociale s'inquiète de l'augmentation de budget qui serait nécessaire pour répondre aux demandes et la responsable du service insertion, en charge de ce dossier, organise des réunions de concertation et entreprend alors un travail de clarification de l'offre de proximité d'une part et de coordination entre financeurs d'autre part.

On observe ici que l'intervention est double, de formalisation de l'action associative d'une part pour la rendre intelligible par les financeurs, de standardisation de cette action d'autre part, les structures associatives étant « accompagnées » pour « répondre à un certain nombre de besoins » (Chargée de mission centre de ressources formations linguistiques missionnée par la Ville). Elle renvoie à certains des éléments forts de la logique de rationalisation budgétaire, en particulier la volonté d'homogénéité et de transparence.

Lien et Proximité, dans son action spécifique, a accepté de recevoir les acteurs municipaux pour expliquer les actions qu'elle menait, mais n'a pas souhaité participer aux réunions de concertation. Dans un premier temps, la directrice s'oppose même à la démarche, ancrée qu'elle est dans une logique d'innovation et d'expérimentation locale « Sous prétexte qu'ils nous donnent des sous, ils vont nous imposer comment on doit travailler et en plus, on va vers une homogénéisation, ce qui est franchement dommage. (...) Je pense que la diversité est une richesse dans le social ». Son attitude change cependant quand elle comprend que cette démarche de formalisation et d'homogénéisation est un passage obligé pour mobiliser des financements : "Mais quelque temps plus tard, j'ai découvert que c'est grâce à ce cadre de référence qu'elle [la responsable du service insertion] a sauvé le financement du FLE (...) » (Directrice Lien et Proximité).

Le cas du FLE à visée sociale montre que la visibilité des actions, propre à la logique de la rationalisation budgétaire, est nécessaire à la création ou à la pérennisation de financements. Elle rencontre des limites dans la mesure où cette visibilité suppose l'homogénéisation des actions sur un territoire pour les rendre comparables et susceptibles d'entrer dans une typologie. Or, les financeurs ne sont pas animés par une seule logique, celle de la rationalisation budgétaire, et ils prennent aussi en compte les avantages d'une relative diversité 
en fonction de contextes micro-locaux, ce qui est propre à la logique de l'innovation. Les interactions financeurs-associations s'inscrivent ainsi dans une forme d'hybridation des deux logiques institutionnelles à l'œuvre, aussi incompatibles que ces logiques puissent paraître au premier abord.

\subsection{Le cas du FLE à visée professionnelle : de l'innovation incrémentale à l'innovation de rupture}

\section{De l'insertion sociale à l'insertion professionnelle}

Le FLE à visée professionnelle s'apparente à l'activité décrite précédemment. Toutefois, il s'en écarte car il conduit à toucher à un des objets même de l'association : passer de l'insertion sociale à l'insertion professionnelle. «Jusqu'à présent, on faisait donc l'apprentissage de la langue comme ça (...) et maintenant on nous impose, si on veut avoir les finances, il faut qu'on le fasse dans la perspective d'un emploi. (...) nous ça nous gêne qu'on nous demande impérativement que ça soit en fonction d'une demande d'emploi. » (Administratrice Lien et Proximité). Il s'agit en effet d'orienter le FLE vers une aide à la recherche d'emploi. L'activité FLE à visée professionnelle existait pourtant de façon confidentielle depuis quelques années au sein de l'association. Elle était née suite à un partenariat local, entre le Relais Emploi du quartier, structure d'accueil et d'accompagnement de demandeurs d'emploi, financée par le FSE et pilotée par les personnes en charge du FSE, et des bénévoles et salariés s'occupant du FLE dans l'association.

Le Relais Emploi incitait certains de ses demandeurs d'emplois à suivre des cours d'apprentissage du français chez Lien et Proximité, et des contacts réguliers ont été créés entre les deux organismes. Un atelier plus spécifiquement destiné à la recherche d'emploi avait été expérimenté, et le Relais Emploi avait alors proposé à l'association qu'ils interviennent dans ses locaux. Une action de FLE à visée professionnelle s'était alors engagée, sans qu'il y ait eu d'incitation financière spécifique : une action innovante, issue d'un partenariat local, et mobilisant les compétences spécifiques de l'association développées en matière de création de liens entre acteurs locaux.

Un changement de comportement des financeurs engendre un nouveau positionnement de l'activité

Le recentrage des financements du FSE sur l'insertion professionnelle a conduit à ce que l'action bénéficie aujourd'hui d'un financement spécifique et soit évaluée au regard de cette spécificité « insertion professionnelle ». On constate donc que le FLE professionnel, qui avait émergé à l'initiative de l'association suite à un contact local avec des personnes du Relais Emploi, devient une activité distincte en raison des modifications de financement. Elle gagne alors en visibilité et conforte son statut d'innovation de rupture : elle crée des liens avec des partenaires spécifiques (le Relais Emploi s'est trouvé être le partenaire naturel quand il s'est agi de mettre en place de façon officielle des activités de FLE à visée professionnelle); les personnes en charge de l'activité développent des compétences spécifiques; l'évaluation de l'activité se fait de manière autonome.

L'histoire de cette activité illustre l'articulation possible entre une logique d'innovation et des opportunités de financement. L'intervention des financeurs fragilise une activité, le FLE à visée sociale, et renforce une activité émergente, le FLE à visée professionnelle, mais sans imposer les modalités de réalisation de l'activité. Le financeur utilise d'ailleurs le 
dispositif de l'appel à projet, moins prescripteur de l'activité qu'un appel d'offres avec cahier des charges. Le financeur n'est pas porteur de l'innovation sociale; mais son intervention a joué dans la dynamique d'innovation en contribuant à mettre en lumière une dynamique sous-jacente qui préexistait.

\section{Les interactions des deux logiques au cours du temps}

Nous avons démarré notre propos en posant la question de l'opposition apparente entre une logique de projet associatif, créatrice d'innovations, et une logique de rationalisation budgétaire, prescriptive, qui s'opposerait au développement d'innovations locales, et qui serait portée par les financeurs. Nous avons alors montré, à travers l'étude de cas présentée, que les relations que les financeurs établissent avec les associations ne répondent pas seulement à une logique de rationalisation budgétaire, et que l'action des financeurs comme des associations conduit à une combinaison des deux logiques. Nous mettons ici en relief les différents facteurs de combinaison des deux logiques.

En premier lieu, nous montrons que les financeurs sont eux-mêmes amenés à combiner les deux logiques, et qu'il existe parmi les financeurs une grande diversité d'action. Dans un second point, nous mettons en lumière le fait que l'analyse doit se faire à partir du système d'acteurs composé de l'association et de ses financeurs, car la dynamique des financements et de l'innovation s'inscrit dans l'interaction au sein de ce système. Enfin, nous montrons que l'interaction entre les dynamiques de financement et d'innovation doit aussi s'analyser dans le temps, selon un modèle non pas linéaire et séquentiel mais tourbillonnaire (Akrich, Callon et Latour, 1988).

\subsection{La diversité d'action des financeurs révèle une diversité de combinaison des deux logiques}

Nous avons constaté ici que le dispositif de financement, en tant qu'instrument de gestion (Chiapello, et Gilbert, 2009; Chemin et Gilbert, 2010) est composé de lignes budgétaires, mais aussi d'orientations politiques : conceptions de ce qu'est l'action sociale et de la place assignée à une association parmi les acteurs opérant sur ce champ.

Pour analyser la diversité d'action des financeurs, nous caractérisons leur démarche en identifiant différentes variables. Nous différencions en premier lieu les démarches de financement à caractère « descendant » (cas de la Caf, et des financeurs européens), un cas où les décideurs sont plus éloignés du territoire local et agissent dans le cadre de démarches d'homogénéisation, et les financeurs qui agissent selon une démarche « ascendante », en concertation avec les acteurs locaux (cas des financeurs liés à la ville en particulier), et qui adaptent souvent leur action aux particularités locales, et seraient donc proches des dynamiques d'innovation.

Toutefois, cette distinction n'explique qu'en partie la nature de l'intervention des financeurs dans les dynamiques d'innovation locale. En effet, une démarche de financement descendante, à condition qu'elle ne fige pas l'activité et la possibilité de composition de liens nouveaux, peut avoir un effet de catalyseur d'innovation (cas du financement du FLE par le FSE). Une démarche ascendante, mettant en avant la 
proximité locale, si elle peut contribuer à faciliter l'innovation sociale, peut aussi se traduire par la mise en place d'un cadre prescriptif qui touche à l'activité, et figer la configuration des relations entre les acteurs (la Ville étant confrontée à ce paradoxe dans le cas du financement du FLE à visée sociale). La souplesse avec laquelle les démarches d'homogénéisation liées à la rationalisation budgétaire sont menées dépend alors des personnes représentant les financeurs sur le terrain, qui sont les vis-à-vis des associations. Le choix du dispositif, appel d'offres ou appel à projet, est également déterminant, l'appel d'offres étant par nature plus prescriptif.

Cette première analyse, si elle donne une indication sur l'impulsion souhaitée par un financeur, n'épuise pas la question de la relation entre logique de l'innovation et logique de la rationalisation budgétaire.

\subsection{L'analyse de la combinaison des logiques au niveau du système association- financeurs}

En effet, le cas analysé ici montre que la perspective est de nature plus interactionniste au sens où c'est l'ensemble du système d'acteurs, association et financeurs qui est à considérer, et non les différents acteurs considérés séparément.

L'association développe une compétence de mise en valeur, à travers les financements, du caractère novateur et légitime d'une innovation. Par exemple, quand une association est à l'origine d'une innovation de rupture, comme dans le cas de la création de la Parlothèque, les compétences semblent relever de la capacité à articuler des acteurs et des compétences locales, mais aussi et surtout à assurer la légitimité de l'innovation aux yeux des financeurs. Elle mobilise des lignes de financement variées qu'elle assemble pour «bricoler » une action cohérente avec son projet innovant, comme dans le cas des activités diverses proposées autour de la Parlothèque ou du FLE à visée sociale. Par ailleurs, l'association peut être amenée à saisir plus massivement des opportunités portées par les financeurs, pour soutenir des innovations qui jusqu'ici étaient peu visibles, plutôt incrémentales. C'est le cas des innovations qui se sont développées autour du FLE professionnel. Quand l'association saisit une opportunité ouverte par une évolution des modes de financements, la compétence principale consiste à repérer ces évolutions, et donc à bien connaître l'environnement institutionnel, et à « traduire » (Callon, 1986) les évolutions institutionnelles en projets et en actions ancrés dans la réalité locale. Dans cette perspective, l'insertion de l'association dans son champ organisationnel, et le travail institutionnel réalisé par ses dirigeants lui donnent une légitimité sur ce champ, favorisant la connaissance de l'environnement et le repérage des opportunités.

Il nous apparaît donc plus pertinent d'analyser la combinaison entre les logiques d'innovation et de rationalisation budgétaire en prenant en considération le système association-financeurs.

\subsection{La combinaison des logiques s'analyse aussi dans la dynamique temporelle}

Sur le plan temporel, la perspective classiquement adoptée jusqu'ici par les chercheurs sur le lien entre financement et innovation a présenté le lien entre association et financeurs de manière séquentielle. On présente l'innovation comme émergeant du projet associatif, 
et devant être reconnue ensuite par les financeurs (Dandurand, 2005). On considère que les associations doivent trouver pour les innovations sociales une forme de reconnaissance institutionnelle, trouver « preneurs au niveau des institutions, des organisations, des communautés » (Chambon et al., 1982).

Toutefois, la dynamique temporelle n'est pas aussi linéaire. L'intervention du financeur, qui procure des ressources à l'association, le conduit parfois à jouer en amont un rôle d'allié (Callon, 1986). L'action des financeurs peut en effet s'interpréter en termes de contribution à la légitimité des acteurs car elle met en visibilité, et donc légitime certains comportements innovants. Ainsi, le financement du FLE à visée professionnelle par le FSE contribue à mettre l'accent sur ce moment de saillie de l'innovation, alors que l'innovation elle-même était en germe, nourrie par la succession d'innovations incrémentales liées au FLE à visée sociale. L'action du financeur s'inscrit alors dans la dynamique d'innovation portée par l'association et contribue à la mise en visibilité de l'action. La Parlothèque, quant à elle, a pu jouir du bénéfice de la nouveauté créatrice, mais c'est par une longue expérience et une série d'expérimentations qu'elle est née, même si elle est présentée à un moment donné comme une innovation de rupture également. Les financeurs interviennent donc dans le cycle innovation de rupture/innovation incrémentale car ils contribuent, à travers des mises en visibilité, à définir le statut des innovations.

Les liens entre dispositifs de financement et innovation sociale nous semblent donc apparaître de manière plus claire dès lors qu'on considère l'innovation comme un processus et non comme un résultat (Le Masson, Weil, Hatchuel, 2006) : l'innovation « en train de se faire », et non dans ses attributs constatés expost. Dans le même esprit, le financement gagne à être considéré non comme une séquence d'action délimitée dans le temps, un moment, mais comme une dynamique en interaction avec le processus d'innovation. On peut alors reprendre la métaphore du modèle tourbillonnaire de l'innovation (Akrich, Callon, Latour, 1988) au sens où l'action des uns et des autres ne s'appréhende pas de manière linéaire. Au contraire les actions s'enchevêtrent, des opportunités sont saisies sans qu'elles aient été prévues lors du démarrage des actions. En considérant le processus d'innovation et les financements dans leur caractère dynamique, nous montrons ici comment l'association et les financeurs réussissent à combiner les deux logiques, qui pourraient être contradictoires, auxquelles ils se trouvent confrontés.

\section{Conclusion}

Les recherches portant sur la coexistence de logiques contradictoires au sein d'un champ organisationnel, et ses conséquences sur les acteurs du champ, ont mis l'accent sur les stratégies des acteurs face aux pressions exercées (Oliver, 1991), les structures mises en place au sein des organisations (Reay et Hinings, 2009), les jeux politiques internes aux organisations pour soutenir l'une ou l'autre des logiques (Pache et Santos, 2010), ou encore les changements d'identité (Haveman et Rao, 2006, Battilana et Dorado, 2010). Nous avons proposé ici de considérer que la coexistence entre ces logiques doit s'appréhender au niveau de groupes d'organisation au sein du champ, à savoir une association et ses financeurs, et non au niveau de l'association ou des financeurs considérés de manière séparée. En effet, l'innovation sociale ne résulte pas seulement d'une série d'opérations 
de conception particulièrement ingénieuses, ni uniquement de la qualité d'un partenariat entre acteurs. Elle est à la conjonction de ces éléments et de la disposition conjoncturelle du système dans lequel elle prend place. C'est au sein du système d'acteurs, dans une dynamique d'évolution, que vont se combiner ces logiques qui, même si elles s'opposent sur de nombreux points, trouvent des formes d'articulation au niveau des interrelations entre les acteurs mentionnés.

Nous proposons également de questionner les modèles séquentiels des relations entre les associations innovantes et les financeurs, où l'on considérait l'innovation sociale comme produite par les acteurs de l'économie sociale et solidaire, et devant ensuite trouver des financements pour être légitimée et conduire à une évolution de normes. Nous montrons que les relations entre financeurs et associations en matière d'innovation s'articulent au sein d'un modèle proche du modèle tourbillonnaire d'innovation. La combinaison de deux logiques institutionnelles a priori antagonistes s'actualise dans ce processus tourbillonnaire multi-acteurs. En particulier, les financeurs n'interviennent pas uniquement en fin de processus, mais peuvent être sollicités à différents moments, notamment à différents moments du cycle innovation incrémentale-innovation de rupture.

\section{Bibliographie}

AKRICH M., CALLON M., LATOUR B., (1988). À quoi tient le succès des innovations? Gérer et comprendre, annales des Mines 12, 14-29.

ALTER N., (2000). L'innovation ordinaire, PUF, Paris.

ALTER N., (2006). On ne peut pas institutionnaliser l'innovation In Klein J.L, Harrisson D., L'innovation sociale, Presses universitaires du Québec, Québec, 139-150.

ATAMER T., DURAND A., REYNAUD E., (2005). Développer l'innovation, Revue Française de Gestion 155, 13-21.

BATTILANA J., DORADO S., (2010). Building Sustainable Hybrid Organizations: the Case of Commercial Microfinance Organizations, Academy of Management Journal 53 (6), 1419-1440.

CALLON M., (1986). Éléments pour une sociologie de la traduction : la domestication des coquilles SaintJacques et des marins-pêcheurs dans la baie de Saint-Brieuc, L'année sociologique 36 (2), 169-208.

CHAMBON J.-L., DAVID A., DEVEVEY J.-M., (1982). Les innovations sociales, PUF, Paris.

CHANAL V., MOTHE C., (2005). Comment concilier innovation d'exploration et innovation d'exploitation : une étude de cas dans le secteur automobile, Revue Française de Gestion 154, 173-191.

CHEMIN C., GILBERT P., (2010). L'évaluation de la performance comme analyseur de la gouvernance associative, Politiques et Management Public 27 (1), 55-78.

CHIAPELLO E., GILBERT P., (2009). La gestion comme technologie économique In P. Steiner et F. Vatin. (Ed) Traité de sociologie économique, PUF, Paris.

CLOUTIER J., (2003). Qu'est-ce que l'innovation sociale? Études théoriques ET0314, Crises, UQAM.

COUR DES COMPTES, (2002). La politique de la ville, rapport public particulier, Journal officiel, Paris.

COUR DES COMPTES, (2007). Gestion des crédits d'intervention de l'État au titre de la politique de la ville, rapport au Sénat, Paris.

DAMON J., (2008). Les politiques familiales, PUF, Paris. 
DANDURAND L.,(2005). Réflexion autour du concept d'innovation sociale, approche historique et comparative, Revue Française d'Administration publique 115, 377-382.

DEMOUSTIER D., (2005). Les associations et leurs partenaires publics, anciens enjeux, nouvelles attentes, Cnaf, Informations sociales 121 (1), 120-131.

DIMAGGIO P., POWELL W., (1983). The iron cage revisited: institutional isomorphism and collective rationality in organizational field, American Sociological Review 48 (2), 147-160.

EPSTEIN R., (2010). Des politiques publiques aux programmes : l'évaluation sauvée par la LOLF? Les enseignements de la politique de la Ville, Revue française des affaires sociales 2010/1-2, 227-250.

FRIEDLAND R., ALFORD R. R., (1991). Bringing Society Back in: Symbols, Practices, and Institutional Contradictions. In W. W. Powell et P. J. Di Maggio. (Ed.) The New Institutionalism in Organizational Analysis. University of Chicago Press, Chicago, 232-266.

GLASER B. G., STRAUSS A., (1967). The discovery of Grounded Theory: Strategies for Qualitative Research, Aldine, Chicago.

GOODRICK E., SALANCIK G.R., (1996). Organizational Discretion in Responding to Institutional Practices: Hospitals and Caesarean Births, Administrative Science Quarterly 41(1), 1-28.

GREENWOOD R., RAYNARD M., KODEIH F., MICELOTTA E.R., LOUNSBURY M., (2011). Institutional Complexity and Organizational Responses, The Academy of Management Annals 5 (1), 317-371.

GREVE H., (2007). Exploration and Exploitation in Product Innovation, Industrial and Corporate Change 16 (5), 1-31.

HAMEL J., (1997). Étude de cas et sciences sociales, l'Harmattan, Paris.

HAVEMAN H.A., RAO H., (2006). Hybrid Forms and the evolution of Thrifts, The American Behavioural Scientist 49 (7), 974.

HEYRAUD E., (2010). La politique de la Ville, maîtriser les dispositifs et les enjeux, Berger-Levrault, Paris.

HILLIER J., MOULEART F., NUSSBAUMER J., (2004). Trois essais sur le rôle de l'innovation sociale dans le développement territorial, Géographie, Économie, Société 6 (2), 129-152.

JARZABKOWSKI P., MATTHIESEN J., VAN DE VEN A., (2010). Doing which Work? À Practice Approach to Institutional Pluralism In T. Lawrence, R. Suddaby and B. Léca (Eds.) Institutional Work: Actors and Agency in Institutional Studies in Organizations, Cambridge University Press, Cambridge, 284-316.

KLEIN J.L., HARRISSON D., (2007). L'innovation sociale - émergence et effets sur la transformation des sociétés, Presses de l'Université du Québec, Québec.

LE MASSON P., WEIL B., HATCHUEL A., (2006). Les processus d'innovation. Conception innovante et croissance des entreprises, Hermès-Lavoisier, Paris.

LÉVESQUE B., (2002). Les entreprises d'économie sociale, plus porteuses d'innovations sociales que les autres? CRISES, Collection Études théoriques, n ET0205, Montréal.

MERTON R.K., (1949/1965). Éléments de théorie et de méthode sociologique, trad. franç, Plon, Paris.

MEYER J., ROWAN B. (1977/1991). Institutionalized Organizations: Formal Structure as Myth and Ceremony In W. W. Powell et P. DiMaggio. (Ed.) The New Institutionalism in Organizational Analysis, The University of Chicago Press, Chicago, 41-62.

NEYRAND. G., (1995). Sur les pas de la Maison Verte. Des lieux d'accueil pour les enfants et leurs parents, Syros, Paris.

OLIVER C. (1991). Strategic Responses to Institutional Processes, Academy of Management Review 16, 145-179.

PACHE A., SANTOS F., (2010). When Worlds Collide: the Internal Dynamics of Organizational Responses to Conflicting Institutional Demands, Academy of Management Review 35 (3), 455-476.

REAY T., HININGS C.R., (2009). Managing the Rivalry of Competing Institutional Logics, Organization studies 30 (6), 629-652.

REYNAUD J.D., (1988). Les régulations dans les organisations : régulation de contrôle et régulation autonome, Revue Française de Sociologie 29 (1), 5-18. 
THORNTON P. H., OCASIO W., (1999). Institutional Logics and the Historical Contingency of Power in Organizations: Executive Succession in the Higher Education Publishing Industry, 1958 to 1990, American Journal of Sociology 105, 801-843.

TUSHMAN M., ANDERSON P., (1986). Technological Discontinuities and Organizational Environments, Administrative Science Quarterly 31 (3), 439-465.

TUSHMAN M., ROMANELLI E., (1985). Organizational Evolution: A Metamorphosis Model of Convergence and Reorientation. Research on Organizational Behavior 38, 1-23.

YIN R.K., (1990). Case Study Research - Design and methods, Sage, Newbury Park. 


\section{Annexe : Note méthodologique}

\section{La collecte des données}

Pour comprendre l'histoire et l'actualité de l'association étudiée, nous avons mené des entretiens $(n=30)$, des observations d'activité $(n=4)$, l'observation d'une conférence du président du Conseil général et collecté des documents (volume 450 pages) auprès de quatre types d'acteurs, rencontrés seuls (entretiens) ou en interaction avec d'autres (observations) : les financeurs de l'association, les dirigeants de l'association, les acteurs opérationnels (bénévoles et salariés) et les bénéficiaires des services de l'association (Tableau 1).

Les deux activités de l'association ont été étudiées selon le même protocole.

Les entretiens semi-directifs s'appuyaient sur un guide, modulé selon les personnes rencontrées. Il était centré sur trois points :

- les liens entre dispositifs de financement et projet associatif;

- les liens entre dispositifs de financement et activités;

- les modifications organisationnelles en lien avec les points précédents.

La documentation de l'association était notamment constituée de dossiers de demande de financement, de documents de fonctionnement interne (fiches d'évaluation du niveau des apprenants de Français Langue Étrangère, documents pédagogiques divers), de rapports d'activités internes et externes, d'un dossier de presse, d'un ensemble de documents dont l'un retraçait l'histoire de la Parlothèque depuis sa création.

La documentation des financeurs comprenait des rapports d'évaluation des dispositifs publics au niveau local et au niveau national, de cahiers des charges relatifs à des appels à projet ou des appels d'offres.

Tableau 1 : Répartition des données recueillies

\begin{tabular}{|c|c|c|c|}
\hline Acteurs & Méthode de collecte & $\begin{array}{c}\text { Nombre et type } \\
\text { de sources }\end{array}$ & $\begin{array}{c}\text { Durée des entretiens } \\
\text { et quantité } \\
\text { d'information collectée }\end{array}$ \\
\hline Financeurs & $\begin{array}{c}\text { Analyse } \\
\text { documentaire }\end{array}$ & $\begin{array}{c}\text { Évaluation du } \\
\text { dispositif politique } \\
\text { de la Ville, Documents } \\
\text { de communication } \\
\text { à destination } \\
\text { des associations. }\end{array}$ & 250 pages \\
\cline { 2 - 5 } & Entretien & $\begin{array}{c}\text { Représentants des } \\
\text { différentes structures } \\
\text { de financement }\end{array}$ & $\begin{array}{c}\text { 9 entretiens d'une durée } \\
\text { moyenne d'1 heure, soit } \\
9 \text { heures d'entretien }\end{array}$ \\
\cline { 2 - 5 } & Observation & $\begin{array}{c}\text { Allocution } \\
\text { du Président } \\
\text { du Conseil général }\end{array}$ & $\begin{array}{c}1 \text { observation } \\
\text { de 2 heures environ }\end{array}$ \\
\hline Dirigeants & Entretiens & $\begin{array}{c}\text { Président, directrice, } \\
\text { trésorier et } \\
\text { administrateurs. }\end{array}$ & $\begin{array}{c}10 \text { entretiens d'une } \\
\text { durée moyenne } \\
\text { d'1 heure, soit } 10 \mathrm{~h}\end{array}$ \\
\hline
\end{tabular}




\begin{tabular}{|c|c|c|c|}
\hline $\begin{array}{c}\text { Opérationnels } \\
\text { (Bénévoles et salariés } \\
\text { de l'association) }\end{array}$ & Entretiens & 4 salariés, 4 bénévoles & $\begin{array}{c}8 \text { entretiens d'une durée } \\
\text { moyenne d'1 heure, } \\
\text { soit } 8 \mathrm{~h}\end{array}$ \\
\hline Bénéficiaires & Entretiens & $\begin{array}{c}\text { Usager de chacun des } \\
\text { deux services } \\
\text { 1 entretien avec un } \\
\text { partenaire de terrain de } \\
\text { l'association }\end{array}$ & $\begin{array}{c}\text { 2 entretiens d'une durée } \\
\text { moyenne d'1h, soit } 2 \mathrm{~h} \\
\text { d'entretien } \\
1 \text { entretien d'1 heure }\end{array}$ \\
\hline $\begin{array}{c}\text { Interactions } \\
\text { opérationnels- } \\
\text { bénéficiaires }\end{array}$ & Observations & $\begin{array}{c}\text { Observation } \\
\text { des activités de } \\
\text { l'association (1 } \\
\text { Parlothèque, 3 FLE) }\end{array}$ & $\begin{array}{c}4 \text { observations de } \\
\text { trois heures environ } \\
\text { chacune soit } 12 \text { heures } \\
\text { d'observation }\end{array}$ \\
\hline $\begin{array}{c}\text { L'association dans } \\
\text { son ensemble }\end{array}$ & $\begin{array}{c}\text { Analyse } \\
\text { documentaire }\end{array}$ & $\begin{array}{c}\text { Dossiers de demande } \\
\text { de financement, } \\
\text { rapports d'activité, } \\
\text { budgets, documents de } \\
\text { gestion interne }\end{array}$ & \\
\hline
\end{tabular}

\section{L'analyse des données}

Les données ont été analysées pendant la collecte. Des documents intermédiaires ont été rédigés et soumis aux dirigeants et salariés de l'association avec lesquels ils ont été débattus. Ils ont validé la majeure partie de la description de l'association et des phénomènes décrits, et leurs remarques ont été intégrées à l'analyse. Tous les chercheurs ont participé à la collecte des données et tous ont analysé la totalité des entretiens et des notes d'observation. Les résultats de la recherche ont été présentés et discutés lors de réunions du laboratoire de recherche et de colloques.

Les observations ont fait l'objet d'une prise de note systématique. Les entretiens ont été enregistrés et retranscrits. Des notes ont été prises pendant et après les observations. Ces éléments ont fait l'objet d'un traitement de données au moyen du logiciel N Vivo. Ce logiciel permet de dématérialiser les transcriptions d'entretiens et les documents codés, mais n'automatise pas le traitement : les catégories sont créées par le chercheur. L'utilisation des données codées est également simplifiée : des sorties thématiques permettent d'obtenir les extraits d'entretiens pour chaque code, les entretiens ou documents d'origine étant indiqués par le logiciel et un retour à ces transcriptions ou documents d'origine pour relire un contexte plus large étant facilité.

Suivant les indications de la théorie enracinée, le codage s'est fait en deux temps : un temps descriptif et un temps explicatif. Dans notre travail, les catégories utilisées par les acteurs sont d'abord mises en évidence pour une restitution des données qui suive au plus près leur pensée. Conformément à la grounded theory, nous avons d'abord utilisé des catégories descriptives dans une démarche inductive, puis dans un second temps seulement des catégories explicatives.

Exemples de catégories descriptives : « perception des partenaires de terrain », « perception du public », « description des dispositifs de financement », « relations financeurs », 
« ritualisation et innovation », « savoir de terrain, savoir théorique », « valeur sociale, valeur économique », «stratégie de l'association». Les catégories explicatives faisaient un lien plus fort avec la théorie, et comprenaient par exemple, «innovation de rupture », « innovation incrémentale », « dispositif de financement fermé », « dispositif de financement ouvert », « autonomie de l'association », « directivité des financeurs ». 
